
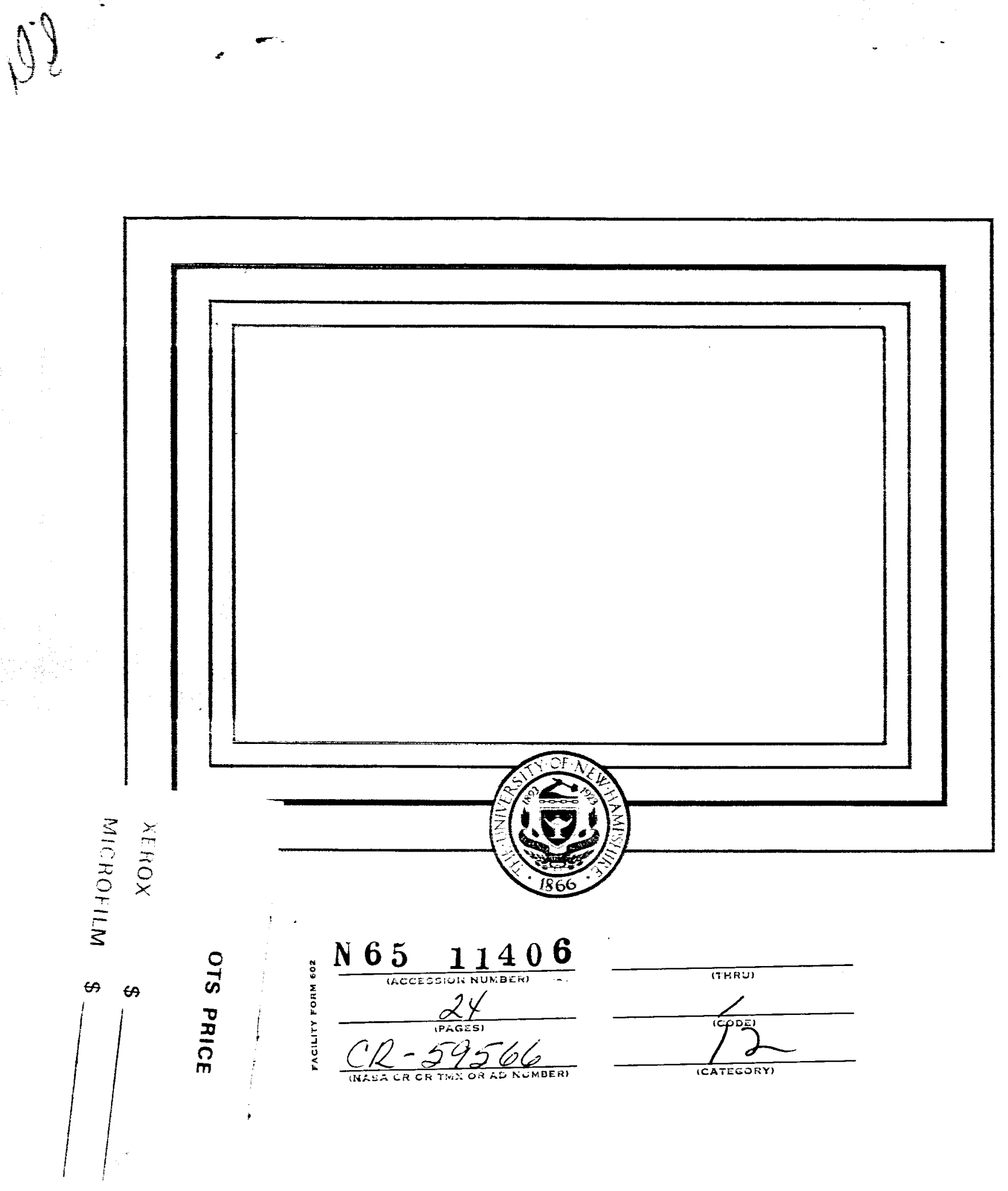

Department of Physics

UNIVERSITY OF NEW HAMPSHIRE

Durham 


\section{Conservation of the First and Second \\ Adiabatic Invariants}

by

Richard L. Kaufmann

Department of Physics

University of New Hampshire

Durham, New Hampshire

September, 1964

This work was supported by the National Aaronautie and

Space Administration under Contract NsG-624. 


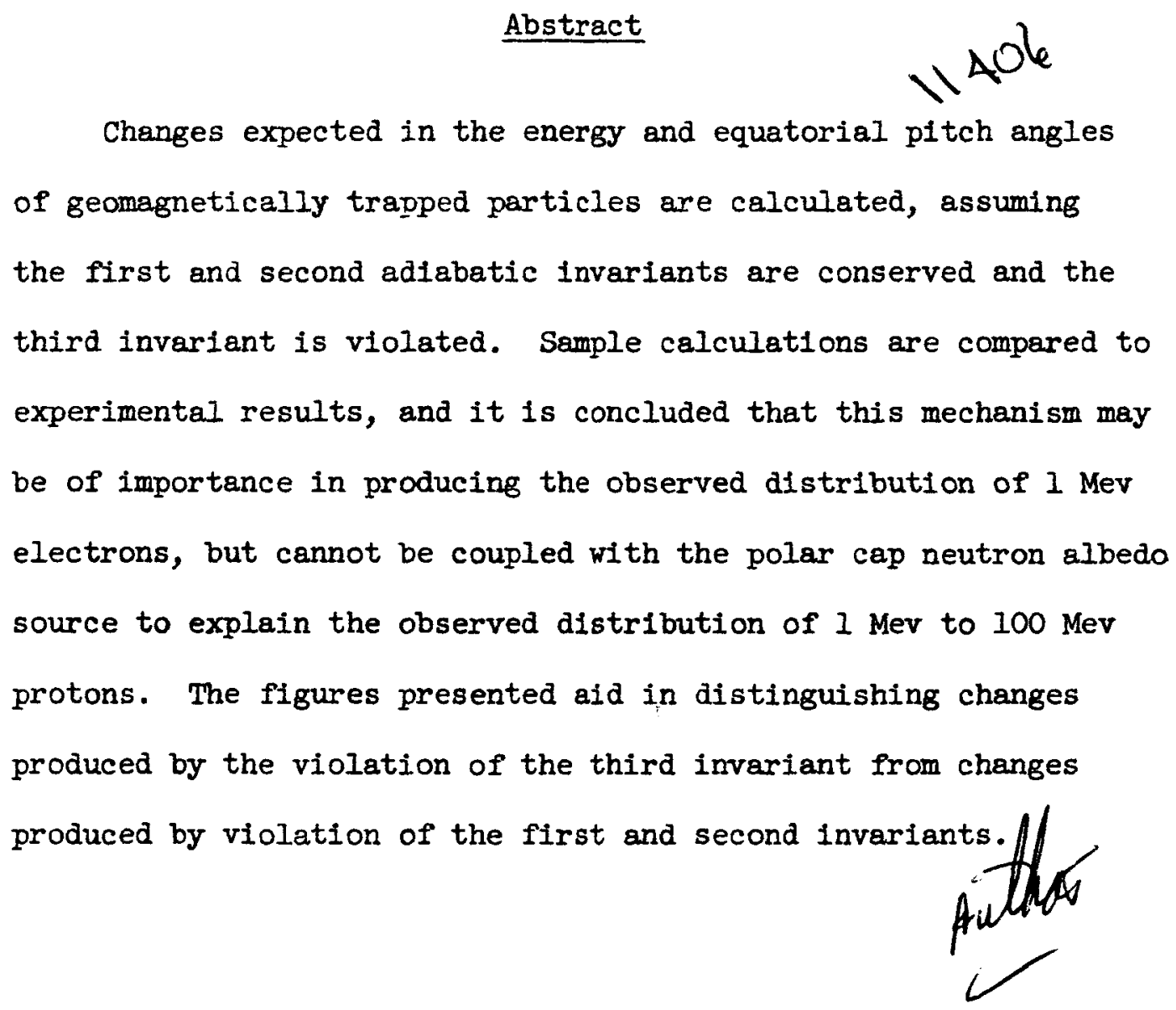




\section{Introduction}

The purpose of the present work is to investigate changes in the energy and equatorial pitch angle (or mirror point) of a particle trapped in a dipole fleld as the particle moves from one magnetic shell to another, assuming the first and second adiabatic invariants (Northrup and Teller, 1960) are conserved. Mechanisms which could produce such motion include breakdown of the third adiabatic 1nvariant by magnetic disturbances (Parker, 1960; Davis and Chang, 1962) and the convection or interchange of entire fleld lines (Gold, 1959). We will not consider speciflc models here, but merely discuss the changes expected as an individual particle moves between magnetic shells. The results are compared with avallable experimental data.

The purpose of studying the breakdown of adlabatic invariants is to aid our understanding of the dynamics of the Van Allen belts. A number of observations suggest that any mechanism which can accelerate trapped particles and also increase their equatorial pitch angles could be of great importance. For example, during the recovery phase of a geomagnetic storm Fan, Meyer, and Simpson (1961), Hoffman, Arnoldy, and Winckler (1962), and Farley and Sanders (1962) observed a large increase in the flux of electrons with energies near $1 \mathrm{Mev}$ and with mirror points concentrated near the equator. Fillius and McIlwain (1964) observed a group of protons with mirror points concentrated near the equator. We will therefore review the consequences of the breakdown of adiabatic invariants with the intention of finding any combinations which will accelerate trapped particles and raise their mirror points. 


\section{Adiabatic Invariants}

The first adiabatic invariant (magnetic moment) is defined as (Northrup and Teller, 1960)

$$
M=\frac{m_{0} \gamma^{2} v^{2}}{2 B}
$$

Where $m_{0}$ is the rest mass, $X$ the relativistic mass ratio, $v_{\perp}$ the component of the particle's total velocity, $v$, perpendicular to the field line, and $B$ the magnetic field strength. We will be interested in cases in which the first and second adiabatic invarlants are conserved, implying that $\gamma$ and $v$ do not change appreciably during one bounce period. It is therefore possible to follow changes in the particle's pitch angle, $\alpha$, during a single bounce period

$$
\frac{2 M}{m_{0} \gamma^{2} v^{2}}=\frac{v_{1}^{2}}{v^{2} B}=\frac{\sin ^{2} \alpha}{B}=\frac{1}{B_{m}}
$$

Where $B_{m}$ is the field strength at the particle's mirror point. Equations 1 and 2 can be combined

$$
M=\frac{m_{0} \gamma^{2} v^{2}}{2 B_{m}}=\frac{m_{0} c^{2}\left(\gamma^{2}-1\right)}{2 B_{m}}
$$

and we will assume this quantity is conserved.

The second adiabatic invariant (integral invariant) is defined as (Northrup and Teller, 1960)

$$
J=\oint m_{0} \gamma v_{11} d s
$$


Where $v_{\| l}$ is the component of the particle's velocity along the field line and ds is an element of are length along the field line. It is sometimes convenient to use a related integral

$$
I=\oint \frac{v_{11}}{v} d s
$$

which depends only upon the field geometry. It is related to $\mathrm{J}$ by

$$
J=\oint\left(m_{0} \gamma v\right) \frac{v_{\|}}{v} d s=m_{0} \gamma v I=m_{0} c I \sqrt{\gamma^{2}-I}
$$

where $J$ and $I$ refer to averages over a single bounce period, and, as noted before, $\gamma$ and $v$ must be essentially constant during a bounce period in order to conserve J. The last forms presented in equations 3 and 6 are most convenient to use at relativistic energies, while the next to last forms presented are more convenient at non-relativistic energies.

We are interested in particle motion in which $M$ and $J$ are conserved, but in which $I, B_{m}$, and $\gamma$ will all vary over time intervals which are long compared to the bounce period.

The integral I has been evaluated numerically (Appendix) and results are presented in Table 1 and Flgure 1 . The quantities $\lambda_{m}, B_{m} / B_{e q}, \alpha_{e q}$, and $R_{e q}$ are also tabulated, where $\lambda_{m}$ is the latitude at which the particle mirrors and $B_{e q}, \alpha_{e q}$, and $R_{e q}$ are the equatorial field strength, pitch angle, and field line radius. The approximate relation

$$
\frac{I}{R_{e q}} \sqrt{\frac{B_{m}}{B_{e q}}}=\left[\begin{array}{lll}
0.280 & \sigma^{-0.552}+0.452 \sigma^{-1.072}
\end{array}\right]^{-1}
$$


Where $\sigma=\left(B_{m}-B_{e q}\right) / B_{e q}$ is shown as the dotted line in Figure 1. This expression is accurate to about $10 \%$ in the range $10^{-3}<\sigma<10^{4}$. For extrapolation of Figure 1 toward the equator $\left(\sigma<10^{-1}\right)$, $I\left(B_{m} / B_{e q}\right)^{I / 2} / R_{e q}=1.480 \sigma$ is a better approximation than is equation 7.

The third or flux invariant is the magnetic flux enclosed by the surface traced out as the guiding center of a trapped particle makes one circuit of the earth.

\section{Breakdown of the Adiabatic Invariants}

Now that the invariants have been defined, it is possible to review the consequences of their violation.

1. If all three invarlants are conserved, there is no acceleration or change in mirror points.

2. If the first invariant breaks down, the second will also generally break down. The third invariant may or may not be conserved. Breakdown of the first invariant requires disturbances with frequencies near the trapped particle's cyclotron frequency, as seen by an observer moving with the trapped particle's guiding center. Wentzel (1962) has suggested that a systematic raising of proton mirror points may be possible in some regions of the magnetosphere as a result of the breakdown of the first and second adiabatic invariants.

3. If the first and third invariants are conserved but the second breaks dowq, acceleration will be accompanied by a lowering of mirror points. Equation 3 shows that when the first invariant is 
conserved, any acceleration requires an increase in mirror point field strength. If the third invariant is conserved, a particle remains on a given magnetic shell, so an increase in mirror point field strength results in motion of the mirror point away from the equator. This mechanism requires disturbances at frequencies near the particle bounce frequency, and has been investigated by Parker (1961).

4. The present work is concerned with the conservation of the first and second invariants and violation of the third. It will be shown in a later section that under these conditions, motion of a particle to a lower field line must be accompanied by acceleration and a motion of the mirror point toward the equator. The required disturbances must have frequencies on the order of the drift frequency.

Violation of the third invariant has been considered by Parker (1960; 1061). He concluded that when this violation is produced by compressions and expansions of the magnetosphere, a given group of particles trapped in the magnetosphere will diffuse so that those moving to lower field lines will gain energy and those moving to higher field lines will lose energy. The net result is a loss of energy from this initial group of trapped particles, i.e. a rather inefficient acceleration mechanism. This mechanism will, therefore, be important only when there is a large source of low energy trapped particles and it is only necessary to explain the production of a much smaller flux of high energy particles. For example, this mechanism could be important in explaining the observed 
flux of I Mev electrons, since some fairly large source of electrons with energies of at least $40 \mathrm{kev}$ must be present on high field lines (0'Brien, 1964$)$.

A number of mechanisms which could cause entire field lines to move within the magnetosphere have been proposed. Gold (1959) first suggested that such motion could be driven by gradients in the plasme energy density. Axford and Hines (1961) described a large convection system which could be driven by a viscous interaction between the magnetosphere and the solar wind. Sonnerup and Laird (1963) investigated the stability of the magnetosphere against spontaneous interchange of field lines. Cole (1964) discussed a diffusion of fleld lines which could be driven by varying electric fields. Any of these mechanisms could carry a group of particles inwards, resulting in a net gain of energy. It is, therefore, of interest to investigate the changes in mirror points of trapped particles as just the third adiabatic invariant is violated.

5. The final possibility is that the first invariant is conserved and both second and third invariants are violated. This requires disturbances near the bounce and drift frequencies. It is possible that particles could be accelerated and their mirror points could simultaneously move toward the equator when only the first invarlant is conserved, but conservation of the first invariant does not require these changes to take place.

To summarize, breakdown of the first, second, and third, of the first and second, of the second and third, or of just the third adiabatic invariant could be accompanied by simultaneous acceleration of charged particles and motion of their mirror points toward the equator. However, only breakdown of just the third invariant must 
necessarily result in acceleration and a systematic motion of mirror points toward the equator as particles move to lower magnetic shells. Breakdown of the other invariants would have to be coupled with some other specific mechanism which would cause mirror points to move toward the equator.

\section{Motion of a Trapped Particle}

Assume a particle is initially trapped in a steady magnetic field and that no electric fields are present, so that all three adiabatic invariants and the energy are constant.

Next a perturbation is applied which causes the third abiabatic invariant and the energy to change and the particle to move to a different magnetic shell. This process will change $\lambda_{m}, B_{m}, R_{e q}$, and $I$, while $M$ and $J$ are conserved. Finally the perturbation stops so that the particle is again trapped in a steady magnetic field with no electric fields present. We wish to find the energy, or $\chi_{2}$, and the mirror point $B_{m 2}$ after the perturbation stops, given the field line, $R_{\text {eq2, }}$, which the particle is finally trapped.

Assuming $M$ and $J$ are conserved, equations 3 and 6 can be combined to give

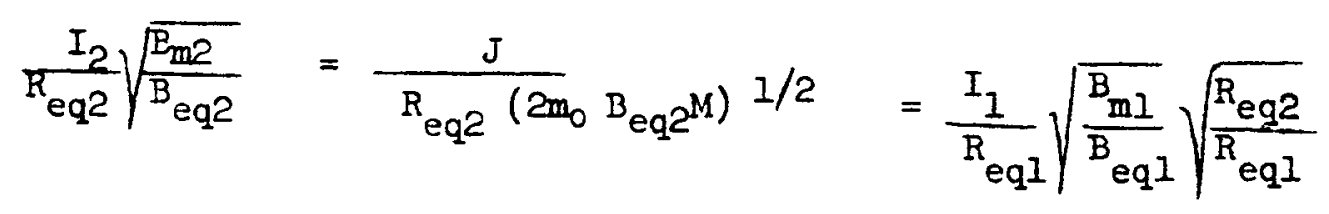

which can be used with figure 1 or equation 7 to find $B_{m 2}$. The energy can then be determined from equation 3.

The first two terms of equation 8 show that a particle's mirror point will move so that $I \sqrt{B_{m}}$ is conserved. Several lines of constant 
8.

I $\sqrt{B}$ are 1 ilustrated in figure 2, where it can be seen that trapped particles will tend to mirror closer to the equator as they drift to lower field lines. Lines of constant field strength are also illus. trated in figure 2. The energy of non-relativistic particles (or $\gamma^{2}-1$ for relativistic particles) is proportional to the mirror point field strength (equation 3). Figure 2, therefore, shows that particles initially mirroring at five or ten earth radii can be accelerated by a factor of several hundred before they strike the dense atmosphere, provided the first and second adiabatic invariants are conserved.

\section{Sample Calculations}

Figure 2 demonstrates that, when the first two invariants are conserved, a particle's mirror point moves toward the equator as the particle moves to a lower field line. In this section, a few sample calculations of the expected changes in equatorial pitch angles will be presented.

Sample calculations were carried out under the assumption that all particles trapped in a thin magnetic shell move inward to another thin magnetic shell. A more realistic calculation would have to be based on a specific mechanism which could produce motion of particles between magnetic shells. Such a mechanism might be more or less efficient at moving particles with mirror points near the equator than those with lower mirror points.

For a sample calculation we first assume particles are produced on the $\mathrm{R}_{\text {eq }}=6$ earth radii field line so that their equatorial pitch angle distribution is isotropic down to some cut-off pitch angle. The particles are then assumed to move to a lower field line, and their new pitch angles are calculated from equation 8 and figure 1. 
The resulting changes in pitch angles are shown in figure 3 for three sample field lines.

Finally, the fluxes expected on several fleld lines are calculated by dividing the $R_{e q}=6$ line into segments and using figure 3 to determine the segment of a lower field line to which the particles initially mirroring in one segment of the $R_{e q}=6$ field line will move. The equatorial flux on the lower field line is obtained from the expression

$$
\Phi_{2}=\Phi_{1}\left(\frac{\Omega_{1}}{\Omega_{2}}\right)\left(\begin{array}{lll}
T_{1} & \cos & \alpha_{e q 1} \\
T_{2} & \cos & \alpha_{e q 2}
\end{array}\right)
$$

where $\Phi$ is the equatorial flux (particles $/ \mathrm{cm}^{2} / \mathrm{sec} / \mathrm{sr}$ ), $\Omega$ is the solid angle subtended at the equator by particles mirroring in a given segment of the field line, and $T$ is the bounce time, which was taken from Hamlin, et al (1961). The quantity ( $\left.T \cos \alpha_{\mathrm{eq}}\right)^{-1}$ is proportional to the time required to move a unit distance along a field line at the equator. The resulting fluxes are presented in figure 4. Fluxes are assumed to drop suddenly to zero at $R=I$ earth radius. Since only the ratio $R_{\text {eql }} / R_{\text {eq2 }}$ enters the calculations, figures 3 and 4 actually give the changes expected in moving between any two field lines where the ratio $R_{\text {eqI }} / R_{\text {eq2 }}$ is $1.5,2$, and 3 , except, of course, the low altitude cutoff would be different.

The equatorial peaking is evident in figure 4. However, even when a shell of particles moves inwards to one third its initial equatorial radius, the ratio of the flux at $\alpha_{\mathrm{eq}}=90^{\circ}$ to that at $a_{\text {eq }}=45^{\circ}$ is seen to increase only by about a factor of 1.6 . 


\section{Comparison with Experiment}

Pitch angle distributions have been calculated by Farley and Sanders (1962) using data from a scintillation counter which detects electrons above a sharp cutoff at $500 \mathrm{kev}$ and from a proportional counter that responds to a broad energy range of electrons, but primarily to electrons with energies on the order of I Mev. Hoffman, Arnoldy, and Winckler (1962) have calculated pitch angle distributions using data from a geiger counter that responds to electrons over a broad energy range, generally near $1 \mathrm{Mev}$. The results reported are that during quiet times the geiger counter observes a fairly broad pitch angle distribution on the $R_{e q}=2.7$ earth radil fleld line, and the distribution becomes progressively more steeply peaked at $90^{\circ}$ pitch angle on the $R_{e q}=3.5$ and 4.4 fleld lines. After a geomagnetic storm the fluxes increase and become more steeply peaked at $90^{\circ}$ pitch angle for the two field lines for which pitch angle distributions are presented $\left(R_{e q}=2.7\right.$ and 3.5). Proportional counter results are available for the $\mathrm{R}_{\mathrm{eq}}=3.3$ field line and are similar to the geiger counter results for $R_{e q}=3.5$. Scintillation counter results for the $R_{e q}=3.3$ field line are also similar after the storm, but before the storm show a minimum at $90^{\circ}$ pitch angle.

These observations can be compared with predictions based on the conservation of the various adiabatic invariants. The relevant frequencies for I Mev electrons mirroring at the equator on the $R_{e q}$ $=3.3$ field line are: cyclotron frequency $=10 \mathrm{kc}$, bounce frequency $=5 \mathrm{cps}$, drift frequency $=.0007 \mathrm{cps}$, or one circuit every 1500 seconds (25 minutes).

If the first invariant is conserved, we have previously argued that breakdown of the second invariant alone cannot produce the observed 
large flux increase and also retain the observed pitch angle distribution peaked at $90^{\circ}$. Therefore, if the first invariant is conserved, the observations can be explained in terms of local acceleration mechanisms only if the third invarlant is violated, i.e. only if I Mev electrons move to lower field lines during magnetic storms. If the electrons observed at $\mathrm{R}_{\mathrm{eq}}=4.4$ can be carried in to $R_{\text {eq }}=3.3$ during a storm, the calculations presented earlier show that individual particles would move so that their equatorial pitch angles would increase. Since even before the storm, electrons at $R_{e q}=4.4$ are strongly peaked at an equatorial pitch angle of $90^{\circ}$, it appears possible that breakdown of the third adiabatic invariant could explain the observed fluxes.

The second possibility is that the first invariant breaks down. If this takes place, then it is not necessary to move electrons inwards across field lines. Either power in the $10 \mathrm{kc}$ frequency range could accelerate electrons and produce equatorial peaking or the required $10 \mathrm{kc}$ disturbance could serve mainly to produce equatorial peaking with little energy change. The acceleration would then be produced by hydromagnetic disturbances in the $10 \mathrm{cps}$ frequency range. The 10 cps signal would accelerate electrons through violation of the second invariant (Parker, 1961) and lower thelr mirror points. The $10 \mathrm{kc}$ disturbance would then be required to raise mirror points more efficiently than the $10 \mathrm{cps}$ disturbance lowers them.

Finally, equatorial peaking has also been reported for protons with energies of a few Mev to about $100 \mathrm{Mev}$ on field lines near $\mathrm{I}=2$. (Fillius and McIlwain, 1964). The relevant frequencies for $10 \mathrm{Mev}$ protons mirroring at the equator are: cyclotron $=60 \mathrm{cps}$, bounce $=$ 1 cps, drift $=.008 \mathrm{cps}$, or one circuit every 130 seconds. 
The most frequently mentioned source of these protons is the decay of polar cap neutrons (Ienchek and Singer, 1963), but such protons do not have mirror points concentrated at the equator. Since protons produced by this mechanism on higher field lines are also peaked well away from the equator, it does not appear possible that the observed peaking could be produced by breakdown of the third invariant. The remaining possibilities are a breakdown of the second invariant (which would be accompanied by deceleration if equatorial mirror points are to be achieved) or breakdown of the first invariant, as discussed In connection with the $1 \mathrm{Mer}$ electrons.

\section{- $\quad \underline{\text { Summery }}$}

The motion of trapped particles was discussed in terms of conservation of the adiabatic invariants. It was found that if the first and second invariants are conserved but the third breaks down, then motion of particles to lower field lines must be accompanied by acceleration and an increase in the particle. 's equatorial pitch angle. The changes expected in the equatorial pitch angle and in the equatorial directional flux were calculated for a sample distribution of trapped particles, and graphs were provided so that similar calculations could be made for other distributions.

Results were compared to measurements of electrons with energies on the order of $1 \mathrm{Mev}$ and protons with energies from 1 to $100 \mathrm{Mev}$. It was concluded that the observed changes in electron fluxes and pitch angle distributions could conceivably be produced by violation of only the third adiabatic invariant. The only other 
possible local acceleration mechanism which could explain the observed results involves the breakdown of the first adiabatic invariant, requiring disturbances near a frequency of $10 \mathrm{kc}$. It was concluded that the observed distribution of 1 to $100 \mathrm{Mev}$ protons could not be produced by decay of polar cap neutrons coupled with breakdown of only the third adiabatic invariant. Breakdown of either the first or second invariant, requiring disturbances with frequencies near 60 cps or 1 cps would be required if the polar cap neutron source is to be of importance.

Violation of the third adiabatic invariant is expected to be of particular importance at high altitudes within the magnetosphere, where large scale field distortions are cormon, and within any convection system which is set up. The figures presented in this paper illustrate the energy and pitch angle changes expected when just the third invariant is violated. 


\section{Appendix}

The integral of equation 5 can be calculated for a dipole field using the equation of a field line

$$
R=R_{\text {eq }} \sin ^{2} \theta
$$

where $R$ is the radial distance from the dipole center and $\theta$ is the angle from the dipole axis.

The field strength along this field line is

$$
B=B_{\text {eq }} \frac{\left(4-3 \sin ^{2} \theta\right)^{1 / 2}}{\sin ^{6} \theta}
$$

For the calculations we have used $\mathrm{B}_{\mathrm{eq}}=\cdot 312 / \mathrm{R}_{\mathrm{eq}}^{3}$ gauss. Equation 2 is used to follow the particle's motion for one bounce period, and with equations 5, 10, and 11 gives

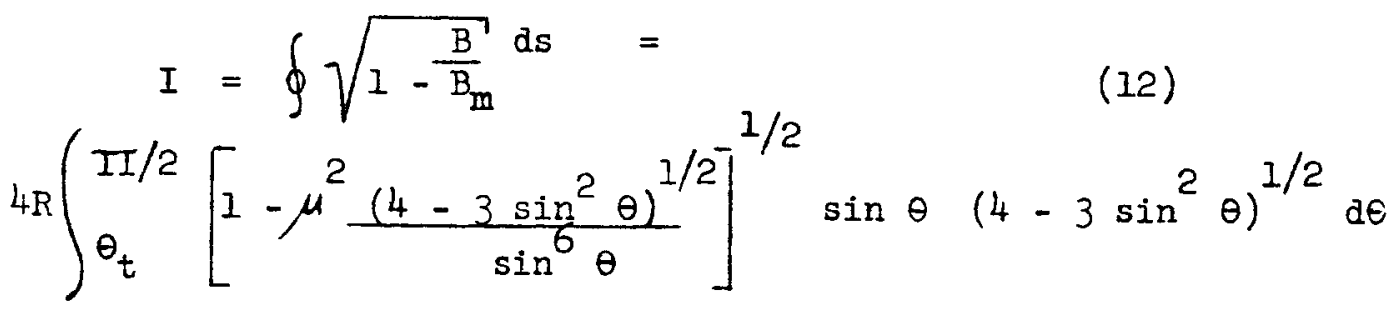

This integral has been evaluated numerically and is presented in table $I$ and figure 1. 


\section{Acknowledgments}

This work was supported by the National Aeronautics and Space Administration under Contract NsG-624. 
1. Axford, W. I., and C. O. Hines, "A Unifying Theory of HighLatitude Geophysical Phenomena and Geomagnetic Storms," Can. J. Phys. 39, 1433-1464, 1961.

2. Cole, K. D., "On the Depletion of Ionization in the outer Magnetosphere during Nagnetic Disturbances," J. Geophys. Research 69, 3595-3601, 1964.

3. Davis, L., Jr., and D. B. Chang, "On the Effect of Geomagnetic Fluctuations on Trapped Particles," J. Geophys. Research 67, 2169-2179, 1962 .

4. Fan, C. Y., P. Meyer, and J. A. Simpson, "Dynamics and Structure of the Outer Radiation Belt," J. Geophys. Research 66, 2607$2640,1961$.

5. Farley, T. A., and N. L. Sanders, "Pitch Angle Distributions and Mirror Point Densities in the Outer Radiation Zone," J. Geophys. Research 67, 2159-2168, 1962.

6. Fillius, R. W., and C. E. McIlwain, "Anomalous Energy Spectrum of Protons in the Earth's Radiation Belt," Phys. Rev. Letters 12, 609-612, 1964.

7. Gold, T., "Motions in the Magnetosphere of the Earth," J. Geophys. Research 64, 1219-1224, 1959.

8. Hoffman, R. A., R. I. Arnoldy, and J. R. Winckler, "Observations of the Van Allen Regions during August and September 1959, 6. Properties of the Outer Region," J. Geophys, Research 67 , $4543-4575,1962$.

9. Lenchek, A. M., and S. F. Singer, "The Albedo Neutron Theory of Geomagnetically Trapped Protons," Planet. Space Sci. 11, 1151-1208, 1963.

10. Northrop, T. G., and E. Teller, "Stability of the Adiabatic Motion of Charged Particles in the Earth's Field," Phys. Rev. 117, 215-225, 1960.

11. Parker, E. N., "Geomagnetic Fluctuations and the Form of the Outer Zone of the Van Allen Radiation Belt," J. Geophys. Research 65, 3117-3130, 1960.

12. Parker, E. N., "Effect of Hydromagnetic Waves in a Dipole Field on the Longitudinal Invariant." J. Geophys. Research 66, 693-708, 1961 .

13. Sonnerup, B. U. Ö., and M. J. Laird, "On Magnetospheric Interchange Instability." J. Geophys. Research 68, I31139, 1963. 


\section{Figure Captions}

Figure 1, The solid line is a numerical evaluation of equation 12. The dotted line is the approximate expression given in equation 7 . Figure 2. Iines of constant $I \sqrt{B}$ in units of (earth radi1). (gauss $^{1 / 2}$ and of $B$ in units of gauss are presented for a dipole field. The lines $I \sqrt{B}$ are traces of a particle's mirror point if the first two adiabat1c invariants are conserved.

Figure 3. The change produced in the equatorlal pitch angle is plotted as a function of initial equatorial pitch angle assuming a particle is initially on the $R_{e q}=6$ earth radii field line, and moves down to Iower field lines while the first two adiabatic invariants are conserved.

Figure 4. Equatorial directional fluxes are presented for a sample distribution of particles. The initial distribution is isotropic on the $R_{e q}=6$ earth radii field line. Fluxes are shown droppling suddenly to zero at $R=1$ earth radius. The curves are arbitrarily normalized at $\alpha_{\mathrm{eq} 2}=45^{\circ}$.

\section{Table Caption}

Table 1. The turning point latitude, $\lambda_{m}$, equatorial pitch angle, $\alpha_{\text {eq, }}$, ratio of the field strength at the mirror point to that at the equator, $B_{m} / B_{e q}$, and the parameter used in equation 7 are tabulated. Numbers in the form $A \pm B$ stand for $A \times 10^{+B}$. 
18.

Table 1

\begin{tabular}{|c|c|c|c|}
\hline$\lambda_{m}$ & $\mathrm{~B}_{m} / \mathrm{B}_{\mathrm{eq}}$ & $\alpha_{e q}$ & $I \sqrt{B_{m} / B_{e q}} / R_{e q}$ \\
\hline $0^{\circ}$ & 1.000 & $90.0^{\circ}$ & 0 \\
\hline 1 & 1.001 & 87.9 & $2.03-3$ \\
\hline 2 & 1.005 & 85.8 & $8.12-3$ \\
\hline 4 & 1.022 & 81.6 & $3.26-2$ \\
\hline 8 & 1.091 & 73.2 & $1.33-1$ \\
\hline 12 & 1.214 & 65.2 & $3.10-1$ \\
\hline 16 & 1.405 & 57.6 & $5.76-1$ \\
\hline 20 & 1.688 & 50.3 & $9.57-1$ \\
\hline 25 & 2.236 & 42.0 & 1.65 \\
\hline 30 & 3.136 & 34.4 & 2.68 \\
\hline 35 & 4.666 & 27.6 & 4.22 \\
\hline 40 & 7.406 & 21.6 & 6.57 \\
\hline 45 & $1.265+1$ & 16.3 & $1.02+1$ \\
\hline 50 & $2.356+1$ & 11.9 & $1.62+1$ \\
\hline 55 & $4.875+1$ & 8.2 & $2.64+1$ \\
\hline 60 & $1.154+2$ & 5.4 & $4.51+1$ \\
\hline 65 & $3.267+2$ & 3.2 & $8.26+1$ \\
\hline 70 & $1.193+3$ & 1.7 & $1.69+2$ \\
\hline 75 & $6.484+3$ & 0.7 & $4.15+2$ \\
\hline 80 & $7.215+4$ & 0.2 & $1.43+3$ \\
\hline
\end{tabular}




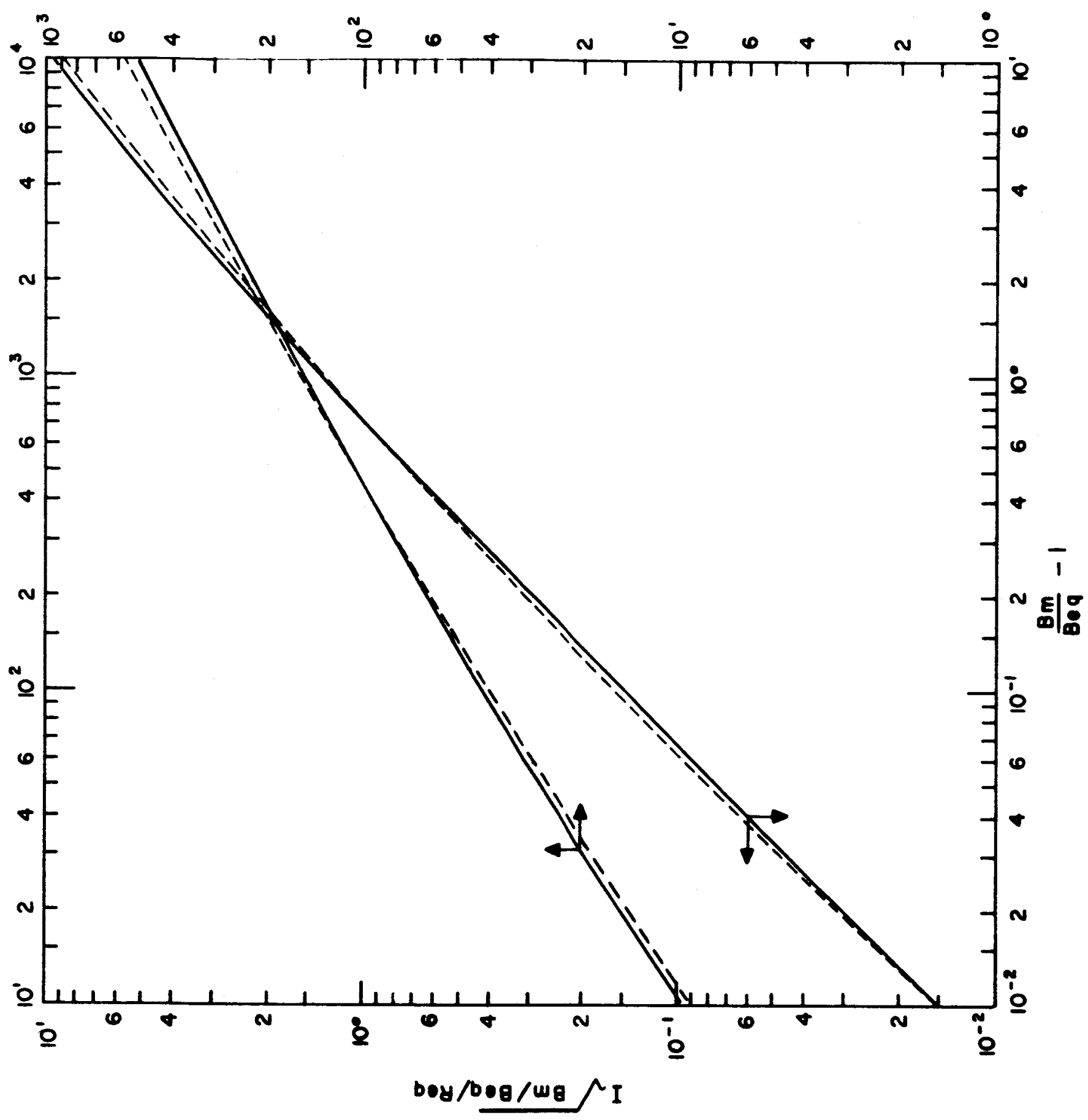




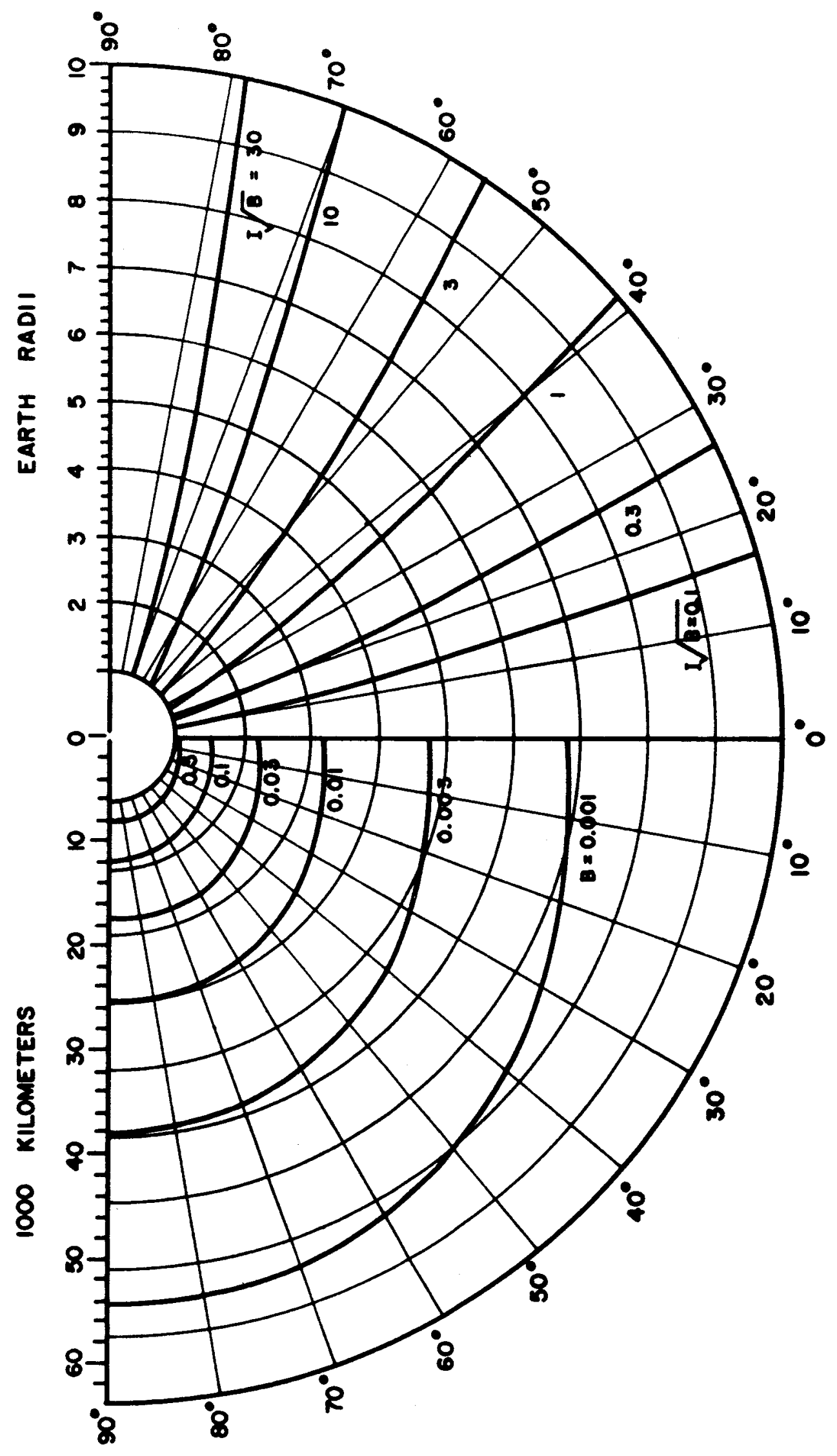




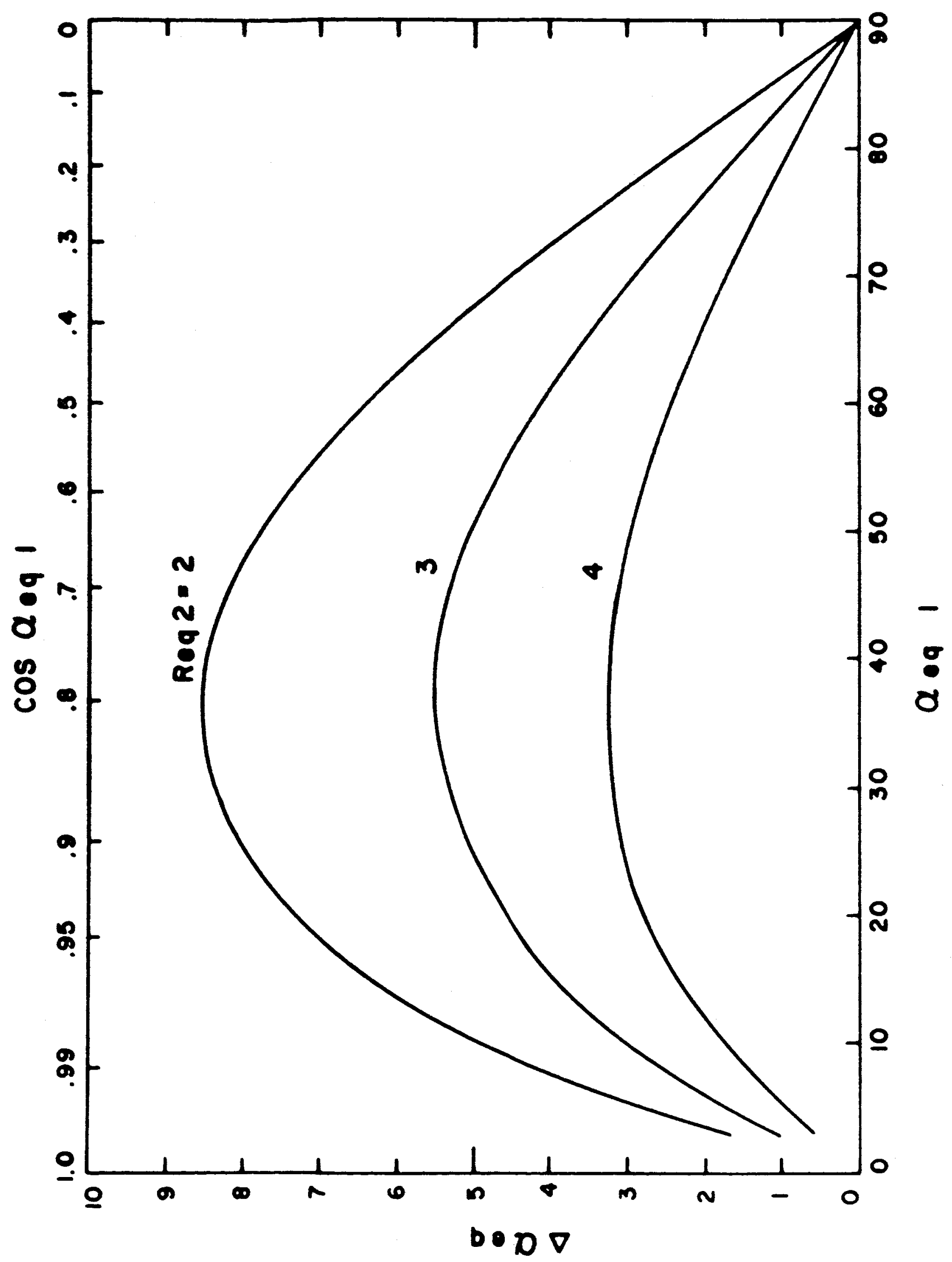




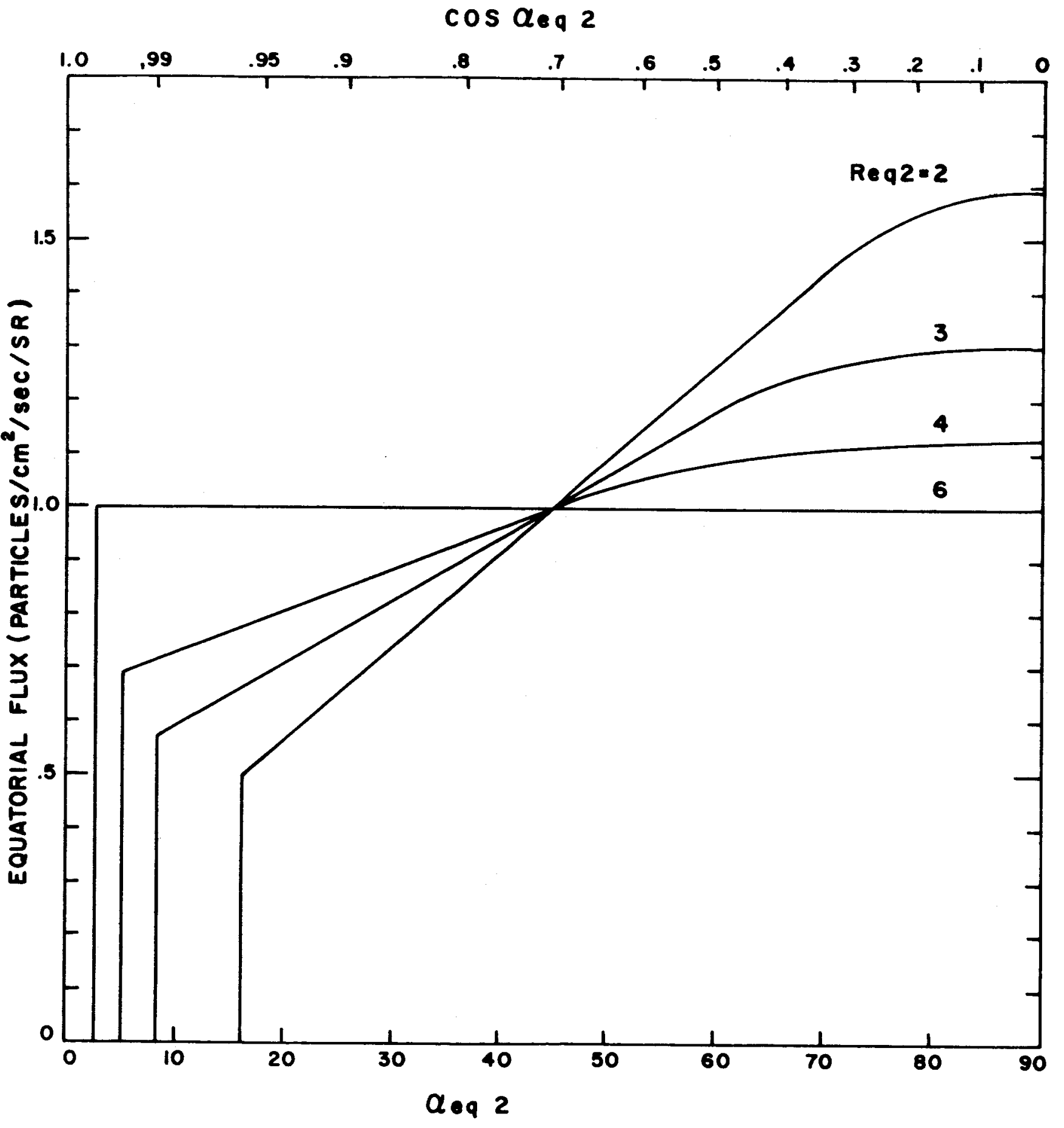

